\title{
Influence of pemphigoid gestationis on pregnancy outcome: A case report and review of the literature
}

\author{
CALIOPIA GAVRIL PARFENE ${ }^{1 *}$, ROXANA ELENA BOHILTEA ${ }^{1-3^{*}}$, \\ BIANCA MARGARETA MIHAI ${ }^{3 *}$, CORINA GRIGORIU ${ }^{2,3}$, IRINA MARGARITESCU ${ }^{4}$, \\ AUREL DORU CHIRITA ${ }^{5}$, CORINA AURELIA ZUGRAVU ${ }^{6}$ and DIMITRIE PELINESCU-ONCIUL ${ }^{1}$ \\ ${ }^{1}$ Department of Obstetrics and Gynecology, Life Memorial Hospital, 012244 Bucharest; \\ ${ }^{2}$ Department of Obstetrics and Gynecology, 'Carol Davila' University of Medicine and Pharmacy, 020021 Bucharest; \\ ${ }^{3}$ Department of Obstetrics and Gynecology, University Emergency Hospital Bucharest, 050098 Bucharest; \\ ${ }^{4}$ Department of Pathology, Onco Team Diagnostic, 030138 Bucharest; ${ }^{5}$ Department of Dermatology, \\ 'Carol Davila' Central Military University Emergency Hospital, 010825 Bucharest; \\ ${ }^{6}$ Department of Food Hygiene and Nutrition, 'Carol Davila' University of Medicine and Pharmacy, \\ 050463 Bucharest, Romania
}

Received June 29, 2021; Accepted July 29, 2021

DOI: $10.3892 /$ etm.2021.10945

\begin{abstract}
Pemphigoid gestationis is considered to be a rare pregnancy exclusive bullous disease, which modifies the course of the pregnancy, with difficulties in the management of the pruritus and skin lesions as well as a possible change in the neonatal outcome. Differential diagnosis of skin lesions and pruritus in pregnancy is challenging, and complementary investigations such as skin biopsy or laboratory tests are indispensable. The correct diagnosis and proper treatment could change the natural course of a pregnancy at risk and could improve maternal and fetal morbidity. We present the case of a patient with pemfigoid gestationis with the aim to highlight: i) the management of this pregnancy-associated skin disorder which transfers this pregnancy into a category of high obstetrical risk pregnancy; ii) the particularities of the course of the pregnancy; and iii) the importance in the differential diagnosis of pregnancy dermatoses. The particularity of this case of pemphigoid gestationis was the acute fetal distress in the absence of intrauterine growth restriction that is frequently found in this pathology, and the management of a rare pregnancy skin condition that currently has no standard treatment.
\end{abstract}

Correspondence to: Professor Roxana Elena Bohiltea or Dr Corina Grigoriu, Department of Obstetrics and Gynecology, 'Carol Davila' University of Medicine and Pharmacy, 37 Dionisie Lupu Street, 020021 Bucharest, Romania

E-mail: r.bohiltea@yahoo.com

E-mail: corigri@gmail.com

*Contributed equally

Key words: pemphigoid gestationis, skin lesions, dermatoses of pregnancy, premature delivery, fetal growth restriction

\section{Introduction}

Pregnancy dermatoses represent a diverse group of mucocutaneous conditions characterized by pruritus and inflammation occurring only during pregnancy or immediately postpartum(1). The most frequent pathologies include atopic eruption of pregnancy, polymorphic eruption of pregnancy (PEP), intrahepatic cholestasis of pregnancy (ICP), pustular psoriasis of pregnancy (PPP) and pemphigoid gestationis (PG). The moment of onset should be noted as there are conditions that occur earlier in pregnancy, such as atopic eruption of pregnancy, and conditions which complicate the second or third trimester of pregnancy or even the postpartum period (PG, PEP and ICP) (2).

$\mathrm{PG}$, in the past known as herpes gestationis, represents an autoimmune bullous disease occurring exclusively after the second part of the pregnancy (3). PG is a rare disease, its incidence being 1 out of 20,000 to 50,000 pregnancies. Besides pregnancy, $\mathrm{PG}$ has been reported to appear in several cases of women with trophoblastic tumors as a paraneoplastic phenomenon (4-6).

The pathogenesis is based on the appearance of circulating IgG1 antibodies against the bullous pemphigoid antigen, known as BP180 or collagen XVII. This antigen is in fact a transmembrane hemidesmosomal glycoprotein, which contributes to the formation of the cutaneous basement membrane zone (7). In PG, the binding of IgG1 to BP180 creates an inflammatory cascade responsible for the detachment of the epidermis from the profound layer, the dermis (8). It appears that the placenta is the initial site of autoimmunity, as these IgG1 antibodies bind also to the amniotic and chorionic epithelia, in addition to the epidermal basement membrane zone, which all have ectodermal origin. One theory sustains the existence of paternal antigens from the second class of major histocompatibility complex on the chorionic villi, which generate an immune response from the mother, resulting in antibodies against the amniotic basement membrane. This theory suggests that these 
antibodies could cross-react with the variable antigens from the maternal skin and induce maternal and even fetal disease (9). Another hypothesis sustains a genetic predisposition, as there have been studies that have shown a possible interconnection between PG and second-class human leukocyte antigen (HLA), especially the phenotype HLA-DR3/HLA-DR4 (10).

Clinical manifestations of PG include extreme pruritus, papules and plaques which form tense blisters that typically appear periumbilical and spread to the trunk and extremities. The lesions usually spare the face and the mucous membranes. Although the symptoms may remit spontaneously before delivery, $75 \%$ of cases burst after delivery and $25 \%$ of cases burst monthly during menses or while using contraceptive pills. Usually, the majority of cases resolve automatically within the first weeks to several months postpartum. PG may reoccur in a more violent form with subsequent pregnancies, but it can also be absent (11).

Regarding neonatal outcome, $\mathrm{PG}$ is associated with a higher risk of premature delivery and, due to a moderate placental failure, women with PG deliver small-for-gestational-age fetuses. There have been no reports of an elevated risk of miscarriage (12). One study concluded that the obstetrical prognosis is influenced by the debut of PG and the existence of blisters on the patient's skin; an early debut in the first or second trimester announces an altered prognosis (13). Although there is not enough evidence to sustain a link between PG and an obscure prognosis, in another study Al-Saif et al reported the outcome of 32 patients with PG, from which 6 were preterm deliveries, 2 pregnancies presented intrauterine growth restriction and another 2 pregnancies finished with abortion or stillbirth (14). IgG autoantibodies could pass the placenta and induce neonatal PG which presents with minor symptoms and resolves spontaneously within weeks. Due to the maternal systemic treatment of the disease, adrenal suppression might occur in babies, but there is a minimum risk even if there were high-dose corticosteroids administered to the mother (15).

The diagnosis is based on the clinical symptoms and signs, skin biopsy with direct immunofluorescence (DIF) and serum level of BP180 antibodies using enzyme-linked immunosorbent assay (ELISA).

As there are few cases of PG, there is yet no standard treatment; the management is mostly based on the clinician's experience combined with the existing literature of bullous pemphigoid and with the collaboration of a dermatologist and a pathologist $(16,17)$. The first recommendation is to use high-potency topical corticosteroids. The second treatment line is represented by systemic corticosteroids, such as prednisone $0.5 \mathrm{mg} / \mathrm{kg}$ daily, which appears to be effective. Postpartum, prednisone can be administered up to $2 \mathrm{mg} / \mathrm{kg}$ daily in severe cases. Other successful postpartum administered therapies include azathioprine, cyclosporine, doxycycline, high-dose intravenous immunoglobulins, cyclophosphamide, rituximab, nicotinamide and immunoapheresis. Oral antihistamines such as chlorpheniramine or loratadine could aid with the control of the pruritus $(18,19)$.

In this article, we present the case of a pregnancy complicated with PG with the aim to highlight the difficulties in the differential diagnosis of pregnancy dermatoses, the management of this particular pathology and the importance of monitoring such a pregnancy with high obstetrical risk.

\section{Case report}

A 40-year-old woman presented to our obstetrics clinic, Life Memorial Hospital (Bucharest, Romania) one year before for early diagnosis and monitoring of a singleton pregnancy. From the gynecologic history, we noted a surgery for a benign condition 2 years prior complicated with an unspecified dermatitis immediately after. Her medical history included the presence of autoimmune thyroiditis. A noninvasive prenatal test was performed and the major chromosomal abnormalities were excluded. The course of the pregnancy was uneventful, and the ultrasound and paraclinical monitoring were normal. We noted nothing abnormal on the blood tests and the fetal growth was according to the gestational age, until the 30th week of pregnancy, when the patient presented with pruritus and papules on the abdomen. The patient was counseled to undergo a dermatologic consultation. The first clinical diagnosis was contact dermatitis and the patient received topical corticosteroids, which had a favorable effect on the remission of the pruritus and cutaneous lesions. After two weeks, the patient returned with generalized and intense pruritus and papules, which raised the suspicion of PG (Fig. 1). Topic corticosteroids administered this time were ineffective and at 35-weeks gestation she receives systemic corticosteroids consisting of 4 doses of $8 \mathrm{mg}$ dexamethasone, as the severity of the symptoms was increasing and the pruritus became unbearable. The skin biopsy performed revealed superficial perivascular dermatitis with lymphocytes and eosinophils, with superficial perivascular lymphocytic and eosinophilic infiltrate and interstitial eosinophilic infiltrate, with some eosinophils in groups located subepidermal (Fig. 2). The direct immunofluorescence showed linear and continuous deposit of $\mathrm{IgG}$ and C3 in the dermoepidermal junction, with no IgA, IgM or C1q deposits (Fig. 3). The existence of a linear deposit of $\mathrm{C} 3$ in the dermoepidermal junction, more specifically at the basement membrane zone, is pathognomonic for PG. Anti BP180 antibodies were not performed, but the skin biopsy, along with the direct immunofluorescence and symptoms confirmed the diagnosis.

At 35 weeks of gestation, due to acute fetal distress, a male fetus of 2,900 $\mathrm{g}$ with an APGAR score at $1 \mathrm{~min}$ of 9 was delivered by Cesarean section. The fetus had a good adaptation and a normal neonatal period, without any pemphigoid lesions.

The patient had an exacerbation of the disease immediately postpartum (Fig. 4) and received topic and systemic corticosteroids, and the evolution of PG was favorable under treatment. The systemic corticosteroids were reduced to a minimum dose with a maximum effect on the remission of the disease. The patient was counseled to use sulphur soap, antihistaminic drugs, fusidic acid and sulfadiazinum for the skin lesions, but all of these increased the rush due to skin dryness; a general recommendation was to avoid ordinary laundry detergent and to replace it with another one for sensitive skin, with no perfume. Immediately postpartum, the patient received $64 \mathrm{mg}$ of methylprednisolone daily and the dose was reduced gradually to a maintenance dose of $32 \mathrm{mg}$ every two days at 3 months postpartum, that proved to be a minimum dose under which the pathology remained under control. The complete remission has not been achieved even 18 months after delivery. 


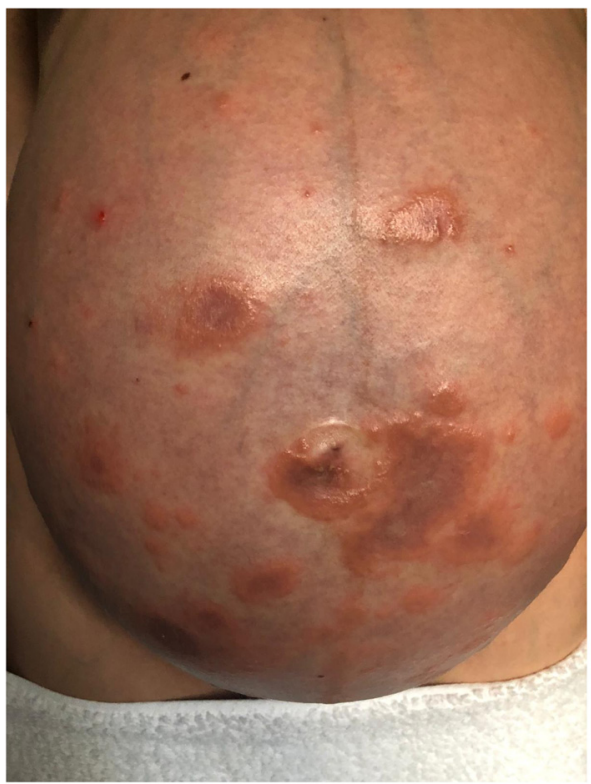

Figure 1. Pemphigoid gestationis lesions occurring in the 32nd week of gestation: urticarial papules and plaques surrounding the umbilicus.

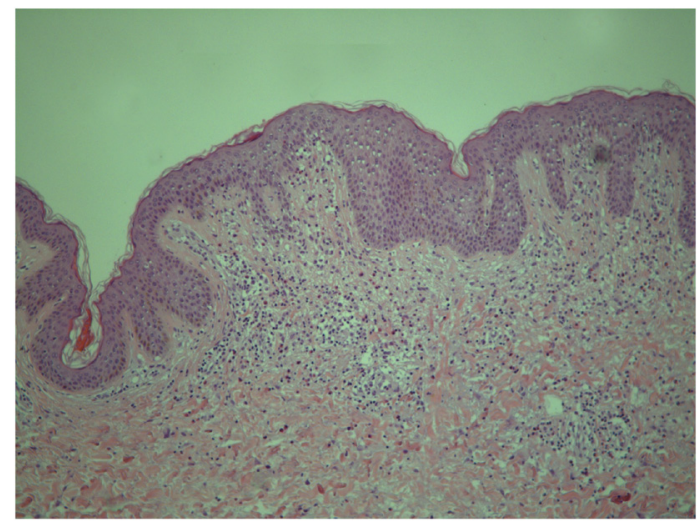

Figure 2. Hematoxylin and eosin (H\&E) staining of the skin biopsy. Conventional $\mathrm{H} \& \mathrm{E}$ histopathological staining shows perivascular dermatitis with lymphocytes and eosinophils, with superficial perivascular lymphocytic and eosinophilic infiltrate and interstitial eosinophilic infiltrate, with some eosinophils in groups located subepidermal (magnification x200).

\section{Discussion}

The particularity of this case of pemphigoid gestationis (PG) consists in the difficulties encountered in the differential diagnosis of pregnancy dermatoses that are able to modify the normal course of the pregnancy and in the atypical complication that imposed iatrogenic prematurity with short- and long-term possible modified fetal prognosis (20). Another particularity of our case were the areas affected by papules and blisters, that atypically included the face, neck, ears and scalp. Any pregnant woman presenting pruritus and skin lesions should be referred to a dermatologist and undergo the necessary investigations, along with the clinical examination of the skin and a detailed medical history. It is of high importance to establish the correct diagnosis so that the patient can receive the optimum healthcare; the evaluation of maternal and fetal prognosis is dependent on the type of dermatoses

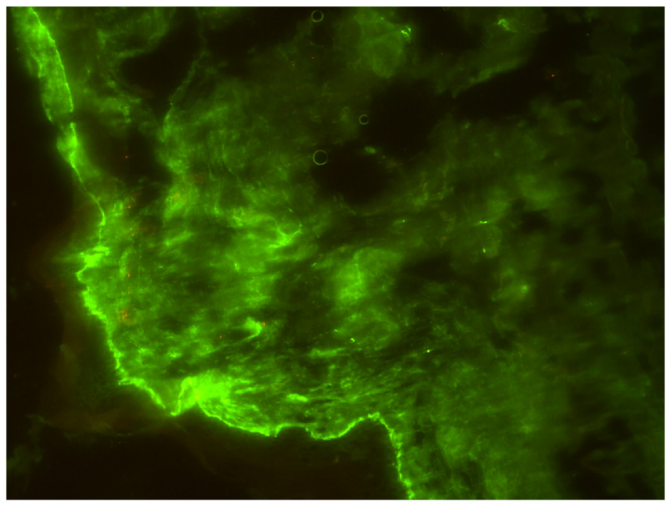

Figure 3. Direct immunofluorescence of the skin biopsy shows linear and continuous deposit of immunoglobulin $\mathrm{G}(\operatorname{IgG})$ and $\mathrm{C} 3$ in the dermoepidermal junction, with no IgA, IgM or C1q deposits (magnification x200).

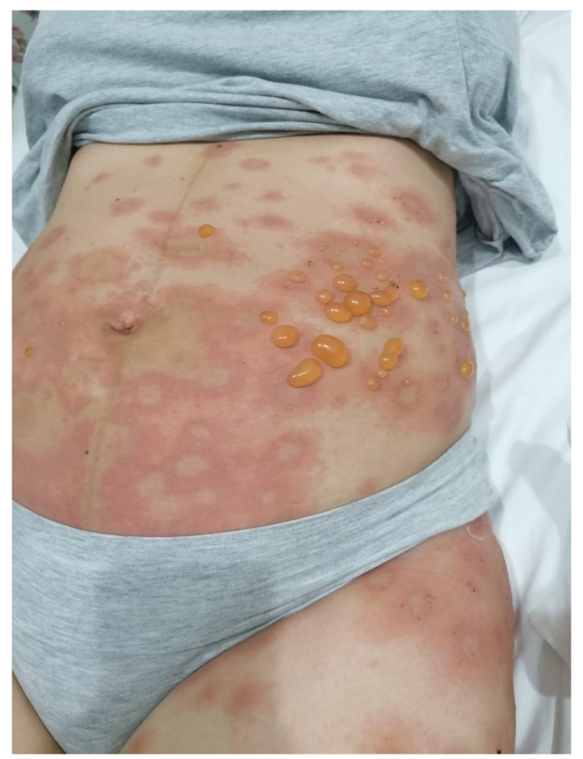

Figure 4. Pemphigoid lesions persistent and exacerbated postpartum: blisters and plaques.

of pregnancy, as polymorphic eruption of pregnancy (5) and atopic eruption of pregnancy $(4,5)$ do not endanger the pregnancy outcome, while pustular psoriasis of pregnancy $(21,22)$, intrahepatic cholestasis of pregnancy (23) and PG (12) could convert the normal uneventful course of pregnancy into a high-risk pregnancy. In order to make the differential diagnosis between intrahepatic cholestasis of pregnancy and PG, the laboratory evaluation should include bile acids, prothrombin time, liver function tests and especially serum anti-BP180 antibodies $(23,24)$. Anti-BP180 antibodies have a specificity and sensitivity between 96 and $100 \%$ for the diagnosis of PG (25). This differential diagnosis is extremely important as the first line treatment for ICP includes ursodeoxycholic acid (26) and for PG consists in topical corticosteroids (16). Furthermore, a skin biopsy, completed with a direct immunofluorescence test, should be performed to differentiate PGs from pustular psoriasis of pregnancy. These two dermatoses present different monitoring of the maternal status. For example, in PG, anti-BP180 antibodies can be used to monitor the course 
of the pathology and the efficiency of the treatment (27) and in pustular psoriasis of pregnancy, a complete metabolic panel should be performed to evaluate electrolyte abnormalities, renal and liver function, possible hypoalbuminemia and the risk for hypocalcemia (22).

In our case, the diagnosis was made considering the clinical features and the result of the skin biopsy. Taking into account the positive diagnosis of $\mathrm{PG}$, the monitoring of the pregnancy was modified as the clinical, blood tests and ultrasound examinations were more frequent. Although, the disease appeared in the third trimester, the fetal growth was not affected despite the intensified activity of the disease and topical and systemic corticosteroid administration. Fetal well-being status was carefully monitored by cerebro-placental ratio and nonstress tests, allowing us to detect the fetal bradycardia in good time.

Fetal myocardium and conduction tissue injury present in systemic lupus erythematosus are due to circulating antibodies to Ro (SSA) and La (SSB), which pass the placenta and affect the normal function of the fetal heart, varying from transient first-degree heart block to complete atrio-ventricular block and hydrops (28). From our knowledge, there are no studies confirming a specific effect of anti-BP180 antibodies on the fetal sinus node or whether these antibodies have a particular affinity for this tissue by a similar mechanism, which implies immune-mediated inflammatory response.

In conclusion, differential diagnosis is the first key to monitoring PG and to ensure the best maternal and fetal outcome. Additionally, there is a real need for further studies concerning the treatment standardization because being a rare cutaneous disease associated exclusively with pregnancy, there is not enough data in the literature to sustain a certain therapeutic attitude and management of this autoimmune pathology.

\section{Acknowledgements}

Not applicable.

\section{Funding}

No funding was received.

\section{Availability of data and materials}

Any additional information concerning the study can be requested from the corresponding author on reasonable request.

\section{Authors' contributions}

CGP and REB conceived the concept of the article after successful management of the presented case to which IM and ADC contributed. BMM, CG and CAZ performed the literature search and wrote the draft of manuscript. CGP and DPO conducted the follow-up of the patient. IM and ADC contributed to the pathological examination of the skin biopsy. CGP, REB, BMM and CG collected, assembled and interpreted the data, making the revision of the manuscript, critically for important intellectual content. All authors have read and approved the final manuscript for publication.

\section{Ethics approval and consent to participate}

The present study was conducted in accordance with the World Medical Association Declaration of Helsinki and was approved by the Institutional Ethics Board of the 'Life Memorial Hospital' (Bucharest, Romania) (approval no. is 24/03.08.2021).

\section{Patient consent for publication}

The patient provided informed consent for publication of the case report.

\section{Competing interests}

The authors declare that they have no competing interests.

\section{References}

1. Ambros-Rudolph CM: Dermatoses of pregnancy-clues to diagnosis, fetal risk and therapy. Ann Dermatol 23: 265-275, 2011.

2. Ambros-Rudolph CM, Müllegger RR, Vaughan-Jones SA, Kerl H and Black MM: The specific dermatoses of pregnancy revisited and reclassified: Results of a retrospective two-center study on 505 pregnant patients. J Am Acad Dermatol 54: 395-404, 2006.

3. Lipozenčić J, Ljubojevic S and Bukvić-Mokos Z: Pemphigoid gestationis. Clin Dermatol 30: 51-55, 2012.

4. Roger D, Vaillant L, Fignon A, Pierre F, Bacq Y, Brechot JF, Grangeponte MC and Lorette G: Specific pruritic diseases of pregnancy. A prospective study of 3192 pregnant women. Arch Dermatol 130: 734-739, 1994.

5. Shornick JK: Dermatoses of pregnancy. Semin Cutan Med Surg 17: 172-181, 1998.

6. Jenkins RE, Jones SA and Black MM: Conversion of pemphigoid gestationis to bullous pemphigoid-two refractory cases highlighting this association. Br J Dermatol 135: 595-598, 1996.

7. Di Zenzo G, Calabresi V, Grosso F, Caproni M, Ruffelli M and Zambruno G: The intracellular and extracellular domains of BP180 antigen comprise novel epitopes targeted by pemphigoid gestationis autoantibodies. J Invest Dermatol 127: 864-873, 2007.

8. KasperkiewiczM,Zillikens D and SchmidtE: Pemphigoiddiseases: Pathogenesis, diagnosis, and treatment. Autoimmunity 45: 55-70, 2012.

9. Sadik CD, Lima AL and Zillikens D: Pemphigoid gestationis: Toward a better understanding of the etiopathogenesis. Clin Dermatol 34: 378-382, 2016.

10. Shornick JK, Jenkins RE, Artlett CM, Briggs DC, Welsh KI, Kelly SE, Garvey MP and Black MM: Class II MHC typing in pemphigoid gestationis. Clin Exp Dermatol 20: 123-126, 1995.

11. Jenkins RE, Hern S and Black MM: Clinical features and management of 87 patients with pemphigoid gestationis. Clin Exp Dermatol 24: 255-259, 1999.

12. Shornick JK and Black MM: Fetal risks in herpes gestationis. J Am Acad Dermatol 26: 63-68, 1992.

13. Chi CC, Wang SH, Charles-Holmes R, Ambros-Rudolph C, Powell J, Jenkins R, Black M and Wojnarowska F: Pemphigoid gestationis: Early onset and blister formation are associated with adverse pregnancy outcomes. Br J Dermatol 160: 1222-1228, 2009.

14. Al-Saif F,Elisa A, Al-Homidy A, Al-Ageel A and Al-Mubarak M: Retrospective analysis of pemphigoid gestationis in 32 Saudi patients-clinicopathological features and a literature review. J Reprod Immunol 116: 42-45, 2016.

15. Aoyama Y, Asai K, Hioki K, Funato M, Kondo N and Kitajima Y: Herpes gestationis in a mother and newborn: Immunoclinical perspectives based on a weekly follow-up of the enzyme-linked immunosorbent assay index of a bullous pemphigoid antigen noncollagenous domain. Arch Dermatol 143: 1168-1172, 2007.

16. Joly P, Roujeau JC, Benichou J, Picard C, Dreno B, Delaporte E, Vaillant L, D'Incan M, Plantin P, Bedane C, et al: A comparison of oral and topical corticosteroids in patients with bullous pemphigoid. N Engl J Med 346: 321-327, 2002.

17. Kirtschig G, Middleton P, Bennett C, Murrell DF, Wojnarowska F and Khumalo NP: Interventions for bullous pemphigoid. Cochrane Database Syst Rev 2010: CD002292, 2010. 
18. Gan DC, Welsh B and Webster M: Successful treatment of a severe persistent case of pemphigoid gestationis with antepartum and postpartum intravenous immunoglobulin followed by azathioprine. Australas J Dermatol 53: 66-69, 2012.

19. Nguyen T, Alraqum E and Razzaque Ahmed A: Positive clinical outcome with IVIg as monotherapy in recurrent pemphigoid gestationis. Int Immunopharmacol 26: 1-3, 2015.

20. Turcan N, Bohiltea RE, Ionita-Radu F, Furtunescu F, Navolan D, Berceanu C, Nemescu D and Cirstoiu MM: Unfavorable influence of prematurity on the neonatal prognostic of small for gestational age fetuses. Exp Ther Med 20: 2415-2422, 2020.

21. Oumeish OY and Parish JL: Impetigo herpetiformis. Clin Dermatol 24: 101-104, 2006

22. Bellman B and Berman B: Skin diseases seriously affecting fetal outcome and maternal health. In: Skin Changes and Diseases in Pregnancy. Harahap K and Wallach RC (eds). Marcel Dekker, Inc., New York, NY, pp129, 1996.

23. Ovadia C, Seed PT, Sklavounos A, Geenes V, Di Ilio C, Chambers J, Kohari K, Bacq Y, Bozkurt N, Brun-Furrer R, et al: Association of adverse perinatal outcomes of intrahepatic cholestasis of pregnancy with biochemical markers: Results of aggregate and individual patient data meta-analyses. Lancet 393: 899-909, 2019

24. Radoi VE, Ursu RI, Poenaru E, Arsene C, Bohiltea CL and Bohiltea R: Frequency of the UGT1A $1 * 28$ polymorphism in a Romanian cohort of Gilbert syndrome individuals. J Gastrointestin Liver Dis 26: 25-28, 2017.
25. Al Saif F, Jouen F, Hebert V, Chiavelli H, Darwish B, DuvertLehembre S and Joly P: French Study Group on Autoimmune Bullous Skin Diseases: Sensitivity and specificity of BP180 NC16A enzyme-linked immunosorbent assay for the diagnosis of pemphigoid gestationis. J Am Acad Dermatol 76: 560-562, 2017.

26. Society for Maternal-Fetal Medicine (SMFM). Electronic address: pubs@smfm.org, Lee RH, Mara Greenberg, Metz TD and Pettker CM: Society for maternal-fetal medicine consult series \#53: Intrahepatic cholestasis of pregnancy: Replaces consult \#13, April 2011. Am J Obstet Gynecol 224: B2-B9, 2021.

27. Huilaja L, Surcel HM, Bloigu A and Tasanen K: Elevated serum levels of BP180 antibodies in the first trimester of pregnancy precede gestational pemphigoid and remain elevated for a long time after remission of the disease. Acta Derm Venereol 95: 843-844, 2015.

28. Srinivasana $\mathrm{S}$ and Strasburger J: Overview of fetal arrhythmias. Curr Opin Pediatr 20: 522-531, 2008. 